\title{
El programa de Educación Maternal en el Sistema Público de Salud en Galicia
}

\author{
The Maternal Education Programme in the Public Health System in Galicia
}

\author{
A Martínez Rodríguez, S Martínez Bustelo, S Pita Fernández, A Ferri Morales, Mª T \\ Conde Regueira
}

\begin{abstract}
Resumen
Objetivos. Los objetivos del presente estudio han sido conocer la presión asistencial de los cursos de Educación Maternal en los centros de salud gallegos, así como su temporalización y las actividades teóricas y prácticas realizadas. Material y métodos. Estudio observacional de prevalencia mediante cuestionario autoadministrado y autofranqueado de todos los Centros de Salud de la Comunidad Autónoma de Galicia que disponen de matrona. El análisis estadístico ha consistido en un estudio descriptivo de todas las variables incluidas en el estudio. En el 81,5\% de las encuestas recibidas se refiere la existencia del Programa de Educación Maternal. En el 93,4 \% de los casos las mujeres que inician el programa lo finalizan. Los contenidos teóricos del programa más frecuentemente desarrollados han sido cuidados en el recién nacido, lactancia y cuidados en el puerperio que están presentes en el $100 \%$ de los casos; y el menos frecuentemente abordado ha sido medicación y embarazo. Los contenidos prácticos del programa más frecuentemente desarrollados han sido las técnicas de respiración (100\%), seguida de la preparación física preparto $(98,1 \%)$; y el menos desarrollado ha sido la recuperación física posparto. El mes de gestación en el que se inició el programa tuvo como mediana 6,5. La duración del programa tuvo como mediana 10 semanas. En el $100 \%$ de las encuestas recibidas se indica que el responsable del programa es la matrona. Según las respuestas recibidas, existe una buena implementación del Programa de Educación Maternal. En cuanto a los contenidos teóricos y prácticos abordados en el período preparto, se observa una gran homogeneidad entre las distintas Unidades, abarcando gran variedad de aspectos. Parece que se enfatiza el programa preparto respecto al posparto, que cuenta con un menor número de horas semanales y de semanas de duración.
\end{abstract}

\begin{abstract}
Aims of the study. The goals of this study have been to know the number of patients who participate in the Maternal Education Program in the Health Centers in Galicia. Timetable, theoretical and practical activities of the program have been analised. Subject and methods. transversal descriptive study. We have sent by post a survey to all the health centers of Galicia which have midwifes. The statistic analyses has been about a descriptive study of all the variables included in the questionnaire. The maternal Education Program exists in the $81.5 \%$ of the replies we have received. In the $93.4 \%$ of the cases, the women both started and finished the program. The theoretical contents more frequently developed have been the medical care for newborn, breast-feeding and medical care in the postpartum period, which are present in the $100 \%$ of the cases. The less frequently developed has been the medication and pregnancy. The practical contents of the program more frequently developed have been the breathe technics $(100 \%)$ followed by physical training during pregnancy $(98.1 \%)$. The less developed topic has been the physical training after delivery. The month of pregnancy when the programme begun had as median 6.5. The lenght of the program had as median 10 weeks. The $100 \%$ of the replies showed that the person in charge of the programme was the midwife. According to the replies we have received, there is a high development of the Maternal Education Program. Related to the theoretical and practical contents taught during the pregnancy, there is so much homogeneity about several aspects among the different centers. It seems to be more emphasis in the prepartum compared to the postpartum program, which has less number of hours per week, and also less weeks of length.
\end{abstract}

\section{Palabras Clave}

Educación para la salud; Embarazo; Período posparto.

Keywords

Health education; Pregnancy; Postpartum period. 


\section{Introduccion}

En el año 2000 según el Instituto Galego de Estadística (IGE) ${ }^{1}$, hubo en dicha Comunidad Autónoma un total de 19.225 partos. La tasa de mortalidad por 100.00 habitantes por embarazo, parto y puerperio fue en dicho año de 0 , al no existir ningún caso.

Sin embargo, las complicaciones del embarazo, parto y puerperio (según datos referidos por el IGE en relación a los enfermos dados de alta) en el año 2000 fueron de 27.655 en Galicia.

En el campo de la mujer embarazada se han realizado diversos programas atendiendo a problemas comunes asociados al embarazo como la incontinencia urinaria o el dolor de espalda ${ }^{2-4}$.

En Galicia la atención a la mujer durante el embarazo y puerperio en el sistema público se aborda fundamentalmente en las Unidades de Psicoprofilaxis Obstétrica en los Centros de Salud.

El presente estudio se ha llevado a cabo con el fin de identificar las características de los programas de educación maternal desarrollados en Galicia. Los objetivos específicos del mismo han sido conocer la presión asistencial de los cursos de Educación maternal en los centros de salud gallegos, las actividades de la enseñanza teórica y práctica así como la temporalización de dichos programas.

\section{Material y métodos}

El estudio se ha efectuado en los centros de salud de Galicia que disponen de matronas en el período comprendido entre los meses de marzo a junio de 2002. Se localizaron un total de 121 Unidades de Psicoprofilaxis Obstétrica ${ }^{5}$. Se trata de un estudio observacional de prevalencia, en el que se han incluido todos los centros de salud de la Comunidad Autónoma de Galicia que entre sus recursos humanos dispusieran de matronas $(\mathrm{n}=121)$.

Como criterio de exclusión se consideró el no querer participar en la encuesta. El número de encuestas respondidas $(\mathrm{n}=65)$, permite estimar los parámetros de interés con una seguridad del $95 \%(\mathrm{a}=0,05), \mathrm{y}$ una precisión de $\pm 12,2 \%$.

A cada centro de salud objeto del estudio según los criterios de inclusión se les remitió un cuestionario autoadministrado y autofranqueado hasta un máximo de tres ocasiones. En dicho cuestionario se recogía información referente al centro de salud, la existencia o no del Programa de Educación MaternalPsicoprofilaxis Obstétrica, el número de años en funcionamiento, la población (habitantes) que cubre su zona básica de salud, el número de partos anuales, el promedio de mujeres que iniciaron y finalizaron dicho programa en el último año. También se incluyó información relativa a los contenidos teóricos y prácticos abordados en el programa y temporalización del mismo tanto en las fases preparto como posparto (tabla 1).

Tabla 1. Contenidos teóricos y prácticos abordados en el programa de educación maternal y temporalización del mismo tanto en las fases preparto como posparto

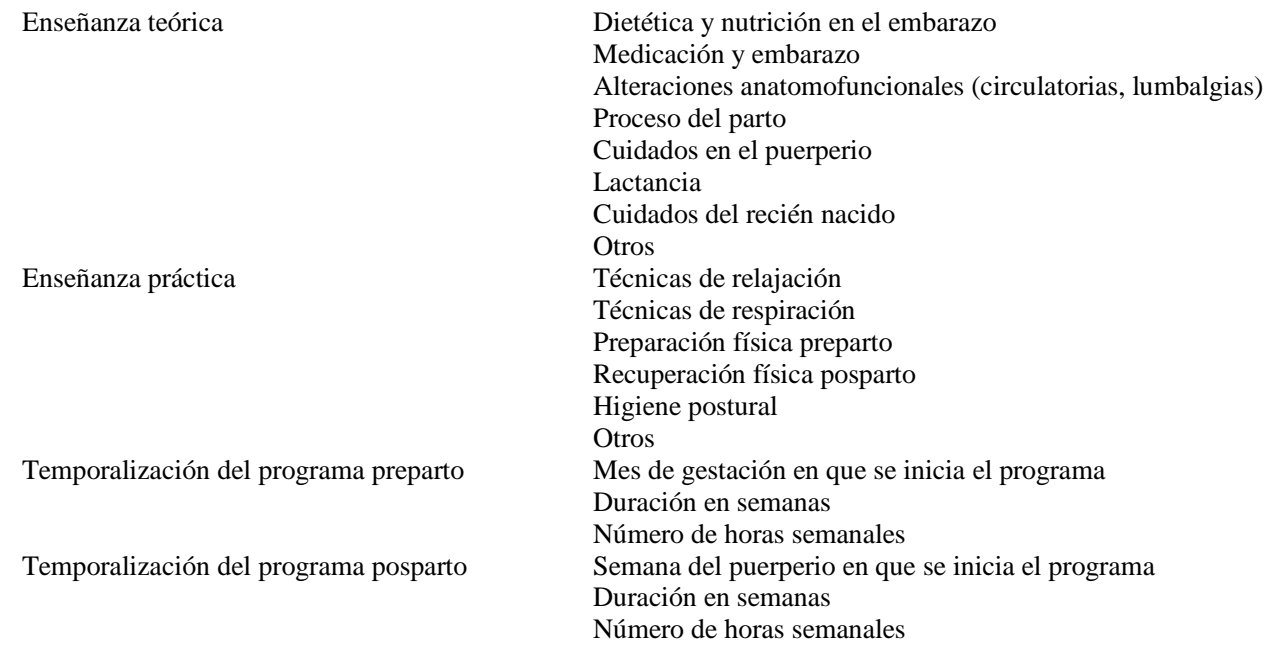

Dietética y nutrición en el embarazo

Medicación y embarazo

Alteraciones anatomofuncionales (circulatorias, lumbalgias)

Proceso del parto

Cuidados en el puerperio

Lactancia

Cuidados del recién nacido

Otros

Técnicas de relajación

Técnicas de respiración

Preparación física preparto

Recuperación física posparto

Higiene postural

Otros

Mes de gestación en que se inicia el programa

Duración en semanas

Número de horas semanales

Semana del puerperio en que se inicia el programa

Duración en semanas

Número de horas semanales 
El análisis estadístico ha consistido en un estudio descriptivo de todas las variables incluidas en el estudio. Las variables cuantitativas se han expresado como media \pm desviación típica. Las variables cualitativas se han expresado como valor absoluto y porcentaje. La asociación de variables cualitativas entre sí, se estimó por medio del estadístico chi cuadrado. La comparación de medias se realizó por medio de la t de Student, test de Mann-Whitney, según procedió tras la determinación de la normalidad de las variables cuantitativas con el test de Kolgomorov-Smirnov. Se consideró estadísticamente significativo valores de $\mathrm{p}<0,05 \mathrm{y}$ todos los procedimientos fueron bilaterales.

Se solicitó el consentimiento a participar de los diferentes centros implicados según los criterios de inclusión y se mantuvo la confidencialidad de la información durante todos los apartados del estudio.

\section{Resultados}

Las Unidades de Psicoprofilaxis Obstétrica según aparece en libro de la Guía de Servicios 2000 editado por la Xerencia de Atención Primaria de la Xunta suponen una cifra de 121 en toda Galicia. Por provincias, es la de A Coruña la que concentra la mayor parte (65) distribuidas en 126 centros de salud. Le sigue a continuación Pontevedra con un total de 92 centros de salud y 43 Unidades de Psicoprofilaxis Obstétrica. Orense y Lugo se sitúan a gran distancia, con 6 y 7 Unidades de Psicoprofilaxis Obstétrica y con 100 y 73 centros de salud, respectivamente. Se realizaron envíos en tres ocasiones a dichos centros de salud que disponían de matronas, con 65 respuestas recogidas $(53 \%)$. En el primer envío se obtuvieron 22 respuestas, de las cuales, siete pertenecían a la provincia de A Coruña, siete a la provincia de Pontevedra, una a la de Orense, y no se obtuvo ninguna de Lugo. También se recogieron 7 encuestas de las cuales no se sabe su procedencia. Tras el segundo envío se recibieron 27 encuestas cubiertas, con la siguiente distribución: 21 pertenecientes a la provincia de A Coruña, una a la de Pontevedra, tres a la de Orense y dos a la de Lugo. Ya, por último, en el tercer y último envío se consiguieron 16 respuestas, 10 de la provincia coruñesa, cuatro de Pontevedra, 0 de Orense, una de Lugo, y otra más sin identificar.

En el 81,5\% de las encuestas recibidas se refiere la existencia de Programa de Educación Maternal. No hay asociación entre la existencia o no del Programa de Educación Maternal y la provincia, a pesar de que el $100 \%$ en Pontevedra refieren existencia de programa y en Lugo sólo lo hacen el 66,7 \%. Los años de funcionamiento de dicho programa oscilan de 1 a 24, con una mediana de 12, no existiendo diferencias significativas entre los años de funcionamiento del programa y las diferentes provincias.

La mediana del número de partos anuales es de 51, y a pesar de que la media de partos anuales es distinta en las 4 provincias no se objetivan diferencias estadísticamente significativas. Entre las que inician el programa y las que lo finalizan hay una diferencia media de 4 mujeres, con una amplitud de 0 a 31. En el 93,4\% de los casos las mujeres que inician el programa lo finalizan (tabla 2). Respecto esta variable tampoco existen diferencias según la provincia.

Tabla 2. Distribución de encuestas según provincia, existencia o no del Programa de Educación Maternal y características de actividad

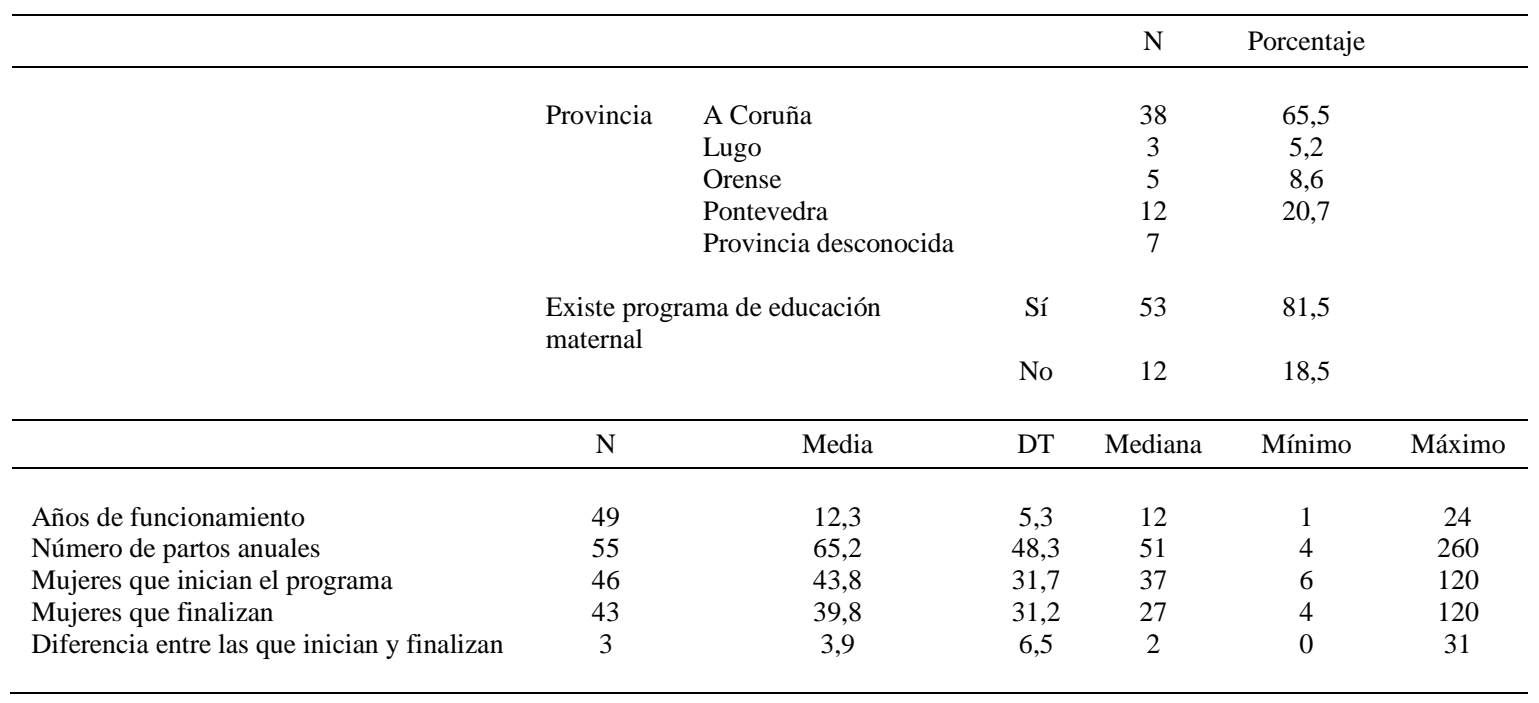


Los contenidos teóricos del programa más frecuentemente desarrollados han sido cuidados en el recién nacido, lactancia y cuidados en el puerperio que están presentes en el $100 \%$ de los casos; y el menos frecuentemente abordado ha sido medicación y embarazo (tabla 3). La presencia o no de diferentes programas es independiente del número de partos o de los años de funcionamiento del programa en los centros. A su vez el número de mujeres que inician el programa no modifica significativamente los contenidos teóricos del mismo.

Tabla 3. Contenidos teóricos del programa que se han realizado

\begin{tabular}{lcc}
\hline & N & Porcentaje \\
\hline & 53 & 100,0 \\
Cuidados en el recién nacido & 53 & 100,0 \\
Lactancia & 53 & 100,0 \\
Cuidados en el puerperio & 52 & 98,1 \\
Proceso de parto & 51 & 96,2 \\
Dietética y nutrición & 46 & 86,8 \\
Alteraciones anatomofuncionales & 40 & 75,5 \\
Medicación y embarazo & 34 & 64,2 \\
Otros temas teóricos & & \\
& & \\
\hline
\end{tabular}

Los contenidos prácticos del programa más frecuentemente desarrollados han sido las técnicas de respiración $(100 \%)$, seguida de la preparación física preparto $(98,1 \%)$; y el menos desarrollado ha sido la recuperación física posparto (tabla 4). La realización de técnicas de relajación es independiente de los años de funcionamiento del programa y del número de partos. La media de mujeres que inician el programa y realizan técnicas de relajación, en comparación con las que no lo realizan, es estadísticamente diferente (46,4 frente a 21,8) (fig. 1). La realización o no de técnicas de respiración así como la preparación física preparto y la higiene postural, son independientes de los años de funcionamiento del programa, del número de partos o de las mujeres que inician el programa. La recuperación física posparto es a su vez independiente de los años del programa y del número de partos, pero hay más mujeres que inician el programa en las que realizan recuperación física posparto que en las que no lo hacen $(51,7$ frente a 32,4), siendo esta diferencia estadísticamente significativa.

Tabla 4. Contenidos prácticos del programa que se han realizado

\begin{tabular}{lcc}
\hline & N & Porcentaje \\
\hline Técnicas de respiración & 53 & 100,0 \\
Preparación física preparto & 52 & 98,1 \\
Técnicas de relajación & 48 & 90,6 \\
Higiene postural & 42 & 79,2 \\
Recuperación física posparto & 30 & 56,6 \\
Otros temas prácticos & 7 & 13,5 \\
& & \\
\hline
\end{tabular}




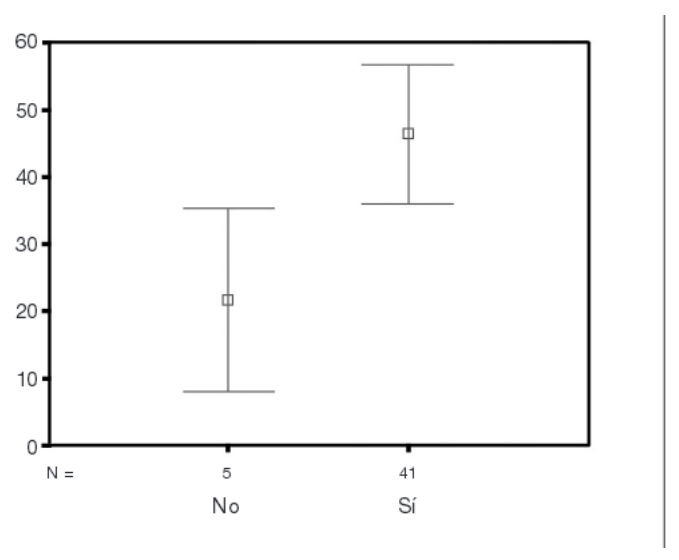

Fig. 1. Media de mujeres que inician el Programa de Educación Maternal y realización o no de técnicas de relajación

El mes de gestación en el que se inició el programa tuvo como mediana 6,5. La duración del programa tuvo como mediana 10 semanas. La semana en la que se inicio el programa posparto así como su duración se muestran en la tabla 5 .

Tabla 5. Temporalización del programa

\begin{tabular}{lcccccc}
\hline & N & Media & DT & Mediana & Mínimo & Máximo \\
\hline Mes de gestación en el que se inicia el programa & 51 & 6,4 & 1 & 6,5 & 1 & 8,5 \\
Duración del programa (semanas) & 50 & 10 & 3 & 10 & 1 & 16 \\
Número de horas semanales preparto & 50 & 2,4 & 2 & 1 & 1 & 6 \\
Semana de inicio del programa posparto & 14 & 4,1 & 4 & 2,2 & 1 & 8 \\
Horas semanales del programa posparto & 14 & 1,6 & 1,5 & 0,5 & 1 & 2,5 \\
Semanas de duración del programa posparto & 10 & 7,6 & 8 & 5,4 & 1 & 16 \\
\hline
\end{tabular}

DT: Desviación típica

En el $100 \%$ de las encuestas recibidas se indica que el responsable del programa es la matrona.

\section{Discusion}

El porcentaje global de respuesta de la encuesta ha sido del $53 \%$, debido fundamentalmente al escaso porcentaje de respuestas obtenido tras el primer envío (18\%) en relación al $40 \%$ esperado ${ }^{6}$.

Las encuestas corresponden fundamentalmente a las provincias de A Coruña y Pontevedra, en donde existen 9,2 y 6,1 veces más de Unidades de Psicoprofilaxis Obstétrica que en Lugo, mientras que la proporción del número de nacimientos en el año 2000 (IGE) es de 3,8 y 3,6 veces mayor en A Coruña y Pontevedra que en Lugo. En relación a Orense la proporción de Unidades de Psicoprofilaxis Obstétrica es de de 10,8 y 7,1 veces menos que en A Coruña y Pontevedra, frente al número de nacimientos en el año 2000 que es de 4,3 y 4,1 veces menos, respectivamente. Esto refleja grandes diferencias en la implantación y desarrollo del Programa de Educación Maternal en las distintas provincias gallegas.

Los datos del estudio muestran que el 93,4 \% de las mujeres que inician el Programa de Educación Maternal lo finalizan, por lo que se aprecia una gran adherencia al mismo.

En cuanto a los contenidos teóricos y prácticos abordados en el período preparto, se observa una gran homogeneidad entre las distintas unidades, abarcando gran variedad de aspectos. Por otro lado, serían necesarios más estudios para averiguar las causas por las que no parece haber relación entre el número de partos y años de funcionamiento del programa con un desarrollo más amplio de los contenidos teóricos del mismo. 
En cuanto a la temporalización, sería recomendable su inicio antes del quinto mes con el fin de prevenir las alteraciones posturales y musculoesqueléticas, tal y como ocurre en otros países como Australia y Reino Unido ${ }^{7,8}$. Además destacamos la gran captación que tienen los programas de Educación Maternal en Galicia, aunque sería interesante realizar futuros estudios para detectar perfiles de no cumplidoras.

Por el contrario la recuperación posparto debería de abordarse en mayor medida, puesto que sólo se lleva a cabo en el $56 \%$ de los casos. En otros países se realizan programas específicos de recuperación posparto, que resultan coste-efectivos, y en los que participan distintos profesionales como el médico, la matrona y el fisioterapeuta ${ }^{3}$. La intervención de este equipo multidisciplinar ha demostrado ser efectiva en la reducción del número de semanas de baja laboral durante el embarazo y en la disminución del dolor lumbar posparto 9,10 .

Si comparamos la temporalización del programa preparto respecto al posparto, se observa una diferencia tanto en las semanas de duración del programa como en el número de horas semanales que se imparten. En este sentido consideramos que se debe ampliar la duración del programa posparto en ambos aspectos. Siguiendo las indicaciones de Roland Leclerc ${ }^{11}$, en el período posparto el número de sesiones debe ser de un mínimo de 10 para garantizar la recuperación de la musculatura del suelo pélvico, la musculatura abdominal y el equilibrio de la columna lumbopélvica.

\section{Limitaciones del estudio}

Al tratarse de un estudio de tipo poblacional los datos se encuentran agrupados, y por tanto en su análisis es factible el haber incurrido en sesgos de información.

\section{Agradecimientos}

A las matronas de los Centros de Atención Primaria del "Servicio Galego de Saúde" (SERGAS).

\section{Bibliografía}

1.Instituto Galego de Estadística. [Fecha de consulta 8 de julio de 2003]. Disponible en: http://www.ige.xunta.es

2.Mason L, Glenn S, Walton I, Hughes C. The relationship between ante-natal pelvic floor muscle exercises and postpartum stress incontinence. Physiotherapy. 2001;12:650-61.

3.Östgaard HC. Assessment and treatment of low back pain in working pregnant woman. Semin Perinatol. 1996;20:61-9.

4.Stuge B, Laerum E, Kirkesola G, Vollestad N. The efficacy of a treatment program focusing on specific stabilizing exercises for pelvis girdle pain alter pregnancy. Spine. 2004;29:351-9.

5.Xunta de Galicia. Guía de servicios 2000. Xunta de Galicia. Consellería de Sanidade e Servicios Sociais;2000.

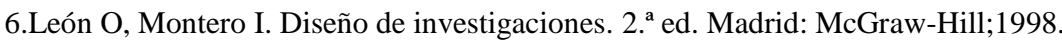

7.Australian Physiotherapy Association and Midwifes Association of Western Education Programme for the childbearing year. 2nd ed. Combined Antenatal Education Committee: Perth, Western Australia;1989.

8.Statement by the Royal College of Midwive.s, the Health Visitor's Association and the Chartered Society of Physiotherapy. Physiotherapy. 1987;73:165.

9.Chiarelli P, Cockburn J. Promotion urinary continence in women after delivery: randomized controlled trial. BMJ. 2002;324:1241-7.

10.Östgaard HC, Roos-Hansson E, Svanberg B. Reduction of back and posterior pelvic pain in pregnancy. Spine. 1994;19: 894-900.

11.Leclerc R. Corps de mère, corps de femme. Paris: Le Cherche Midi;2004 